\title{
High-mobility InSb epitaxial films grown on a GaAs (001) substrate using liquid-phase epitaxy
}

\author{
V. K. Dixit, Bhavtosh Bansal, V. Venkataraman, and H. L. Bhat ${ }^{\text {a) }}$ \\ Department of Physics, Indian Institute of Science, Bangalore 560012, India \\ G. N. Subbanna \\ Materials Research Centre, Indian Institute of Science, Bangalore 560012, India
}

K. S. Chandrasekharan and B. M. Arora

Tata Institute of Fundamental Research, Homi Bhabha Road, Mumbai 400005, India

\begin{abstract}
The growth of epitaxial InSb layers on highly lattice-mismatched semi-insulating GaAs substrates has been achieved via traditional liquid-phase epitaxy. Scanning and transmission electron microscopy show sharp interfaces even at $35 \mathrm{~nm}$ resolution. High-resolution x-ray diffraction studies reveal reflections even up to $2 \theta=153^{\circ}$ with distinct layer and substrate peaks, indicating structural coherence. The films grown were $n$ type and the highest electron mobility obtained was $3.96 \times 10^{4} \mathrm{~cm}^{2} / \mathrm{V} \mathrm{s}$ at room temperature. The band gap varies from 0.17 to $0.23 \mathrm{eV}$ in the temperature range of $300-10 \mathrm{~K}$ and is consistent with the expected variation. These results indicate that the films grown are comparable to those grown by other sophisticated techniques in terms of structural, optical and electrical properties.
\end{abstract}

The high-electron mobility and narrow band gap of InSb make it an important material for high-speed devices, ${ }^{1,2}$ magnetic sensors ${ }^{3}$ and infrared (IR) detectors. For application in electronic and optical devices, InSb thin layers must be grown on semi-insulating IR transparent substrates. CdTe is the only semi-insulating lattice-matched substrate for InSb, but it is very difficult to prevent $\mathrm{In}_{2} \mathrm{Te}_{3}$ precipitate formation at the interface during growth. ${ }^{4,5}$ Hence, $\mathrm{Si}, \mathrm{GaAs}$, and $\mathrm{InP}$ have been used as substrates for InSb epitaxial growth. ${ }^{6-8}$ Even with the large (14.6\%) lattice mismatch, there are many reports on the growth of $\mathrm{InSb} / \mathrm{GaAs}$ heterostructures by molecular beam epitaxy (MBE) $)^{9,10}$ and metalorganic chemical vapor deposition (MOCVD). ${ }^{11,12}$ Although traditionally liquid phase epitaxy (LPE) has been used extensively to grow various heterostructures, the growth of InSb by LPE is restricted due to its low melting point and low growth temperature. Other than a few early papers on homoepitaxy ${ }^{13,14}$ there is no report of the successful growth of InSb/GaAs by LPE. However, the fact that LPE is a simple, inexpensive and widely used growth method is motivation enough to evolve optimized parameters for the growth of high quality $\mathrm{InSb}$ epilayers by this technique on a highly lattice-mismatched substrate as GaAs. In this letter we report LPE growth of $\mathrm{InSb} / \mathrm{GaAs}$ whose structural, transport and optical properties are comparable to those obtained from MOCVD, MBE, etc.

Growth was carried out on (001) semi-insulating GaAs substrates $(1 \mathrm{~mm}$ thick) in a boat-slider type LPE unit, designed and fabricated in our laboratory. ${ }^{15}$ Prior to growth, the GaAs substrates were cleaned using $1 \%$ bromine in methanol at room temperature and then washed in methanol. An indium solution saturated with previously synthesized InSb was used. The optimized III/V mass ratio used for the growth

\footnotetext{
a) Author to whom correspondence should be addressed; electronic mail: hlbhat@physics.iisc.ernet.in
}

was $\sim 3.45$. All materials were of purity better than $5 \mathrm{~N}$ and were obtained from Sigma Aldrich, U.S. In the present investigation we used a ramp cooling routine because a continuous decrease in temperature can provide a driving supersaturation $\beta \propto \Delta T / T_{g}$ at every point during growth and induce orderly epitaxial growth ${ }^{16}$ (where $\Delta T$ denotes supercooling of the solution and $T_{g}$ the initial growth temperature). The films were grown under varied conditions of supersaturation by changing the ramp rate and temperature range. The optimum ramp rate was $0.2^{\circ} \mathrm{C} / \mathrm{h}$ for growth temperature range of $2{ }^{\circ} \mathrm{C}$ and $T_{g}=423^{\circ} \mathrm{C}$. The composition of the films grown was $\mathrm{In}: \mathrm{Sb}=50.53: 49.47$ at. \%, found from energy dispersive $\mathrm{x}$-ray analysis (EDAX).

High-resolution $\mathrm{x}$-ray diffraction (HRXRD) was used to determine the orientation and crystallinity of the films. A $\phi$ scan of an asymmetric reflection was performed initially to check the single-crystal nature of the layer. For this, the goniometer $\omega / 2 \theta$ settings were fixed for the (115) peak of the InSb layer, making an angle of about $15^{\circ}$ with the sample's surface (001). As seen in Fig. 1(a) the scan over $360^{\circ}$ azimuths gave four distinct layer peaks separated by $90^{\circ}$ intervals with negligible scattering in between. Subsequently, several reflections, both symmetric and asymmetric, were recorded. The distinct layer and substrate peaks, separated by several degrees in each reflection, were observed even up to the highest scattering angle of $2 \theta \approx 153^{\circ}$, indicating structural coherence. The peak separation $(\Delta \omega)$ varied from about $4.5^{\circ}$ to $18^{\circ}$ for different reflections. In Fig. 1(b) is displayed, on a relative degree scale, the symmetric (004) reflection, at four azimuths, separated $90^{\circ}$ from each other. From the small difference in $\Delta \omega$ at the four azimuths, the tilt angle $T_{l, s}$ of the epilayer to the substrate was estimated by using the relation,

$$
T_{l, s}=\frac{1}{2}\left[\left(\Delta \omega_{1}-\Delta \omega_{3}\right)^{2}+\left(\Delta \omega_{2}-\Delta \omega_{4}\right)^{2}\right]^{1 / 2},
$$



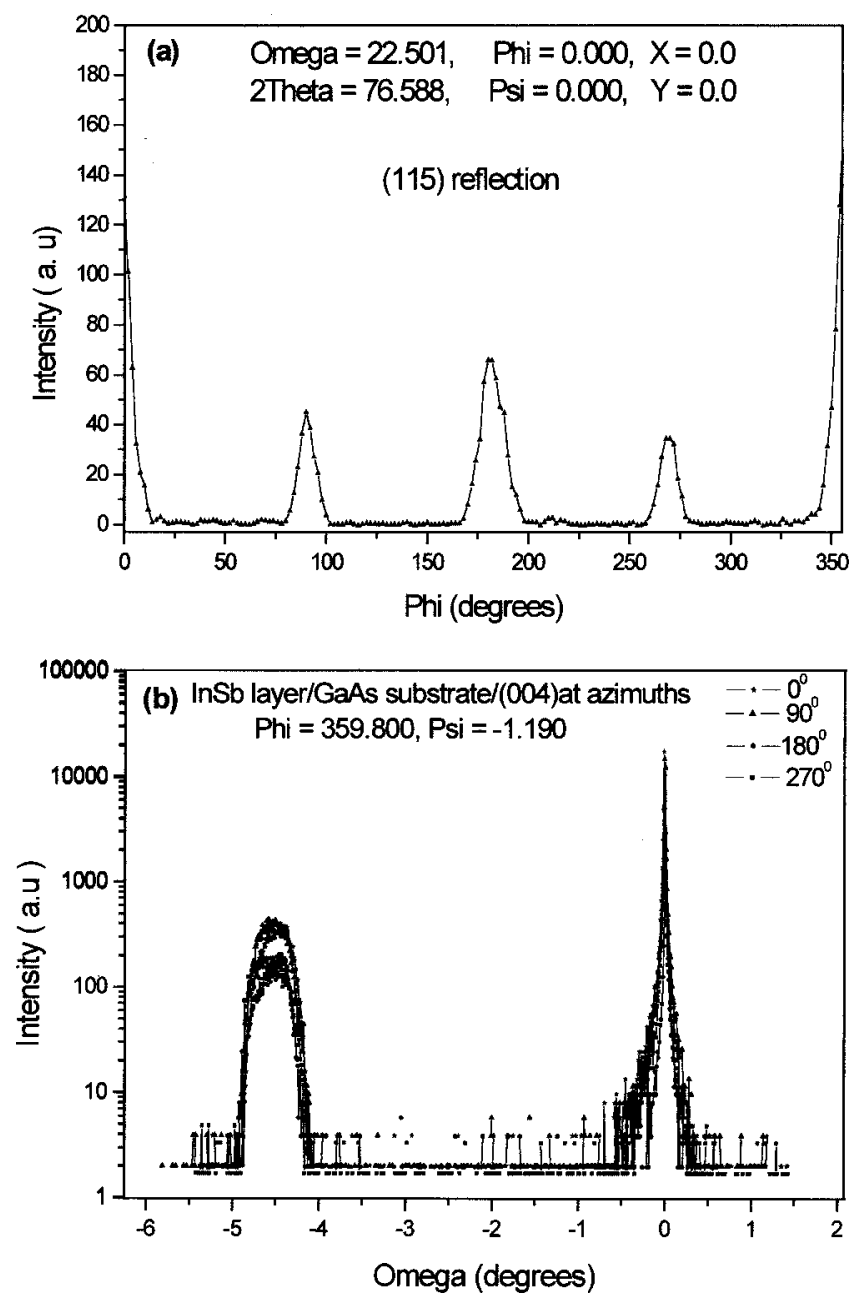

FIG. 1. (a) X-ray diffraction pattern of ( $\phi$ scan) the InSb layer in asymmetric reflection (115) over the $360^{\circ}$ azimuth and (b) representative $\omega$ scans of the InSb layer/GaAs substrate for (004) reflections at four azimuths (film thickness $7 \mu \mathrm{m}$ ).

where $\Delta \omega_{i} s$ are the peak separations at $90^{\circ}$ intervals. The tilt was found to be quite small and was about $0.01^{\circ}$.

The cross section of the film (cleaved sample) observed under a scanning electron microscope (SEM), shown in Fig. 2(a), clearly reflects the sharpness of the interface between the InSb layer and the GaAs substrate. During scanning electron microscopy, simultaneous elemental identification was carried out with (EDAX) to confirm that the region scanned was indeed the InSb/GaAs interface. Cross sectional specimens were prepared and examined at much higher magnification using the JEM-200CX transmission electron microscope (TEM) operating at $200 \mathrm{kV}$. The bright field cross sectional TEM image, shown in Fig. 2(b), indicates a sharp interface with very small coalescing islands [marked $\mathrm{C}$ in Fig. 2(b)]. The selected area electron diffraction pattern from the TEM image of an InSb/GaAs interface is shown in Fig. 2(c). The diffraction spots occur in pairs, corresponding to InSb and GaAs, respectively, indicating that the layer and the substrate are epitaxially oriented.

The temperature dependence of the mobility and carrier concentration in two InSb/GaAs samples, labeled $\mathrm{S} 1$ and $\mathrm{S} 2$, of thickness 3 and $7 \mu \mathrm{m}$, respectively, is shown in Fig. 3. The data reported are representative of the five samples studied. The samples were $n$ type in the entire temperature range.
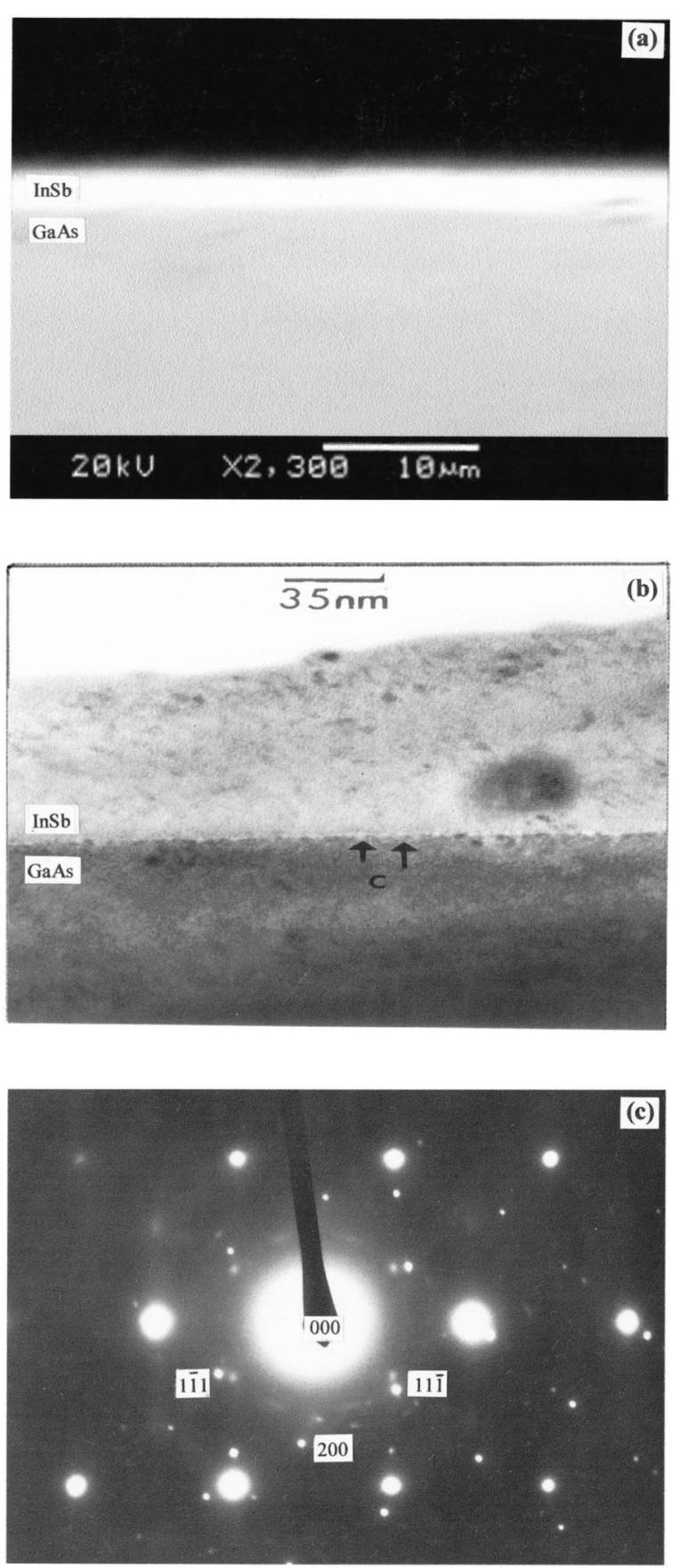

FIG. 2. (a) Scanning electron micrograph of the InSb/GaAs interface, (b) bright field image of the cross section of InSb/GaAs and (c) selected area diffraction pattern of (b).

The highest room temperature (RT) mobility was measured to be $39600 \mathrm{~cm}^{2} / \mathrm{V} \mathrm{s}$, which is comparable to the mobilities reported for InSb/GaAs samples grown by $\mathrm{MBE}$ and MOCVD, ${ }^{10,17-20}$ especially when the purity of the starting material is considered. ${ }^{21}$ Above $350 \mathrm{~K}$, the samples become intrinsic. At $10 \mathrm{~K}$, the carrier concentration indicates a background doping of around $5 \times 10^{16} \mathrm{~cm}^{-3}$. The purity of the materials used for growth has a strong correlation with the background doping and mobility. Initially, when less than 5 $\mathrm{N}$ indium and antimony was used, the carrier density was measured to be more than $1 \times 10^{18} \mathrm{~cm}^{-3}$ and the RT mobility less than $8000 \mathrm{~cm}^{2} / \mathrm{V}$ s. But even for a background doping of $5 \times 10^{16} \mathrm{~cm}^{-3}$, the mobilities around $100 \mathrm{~K}$ are still 


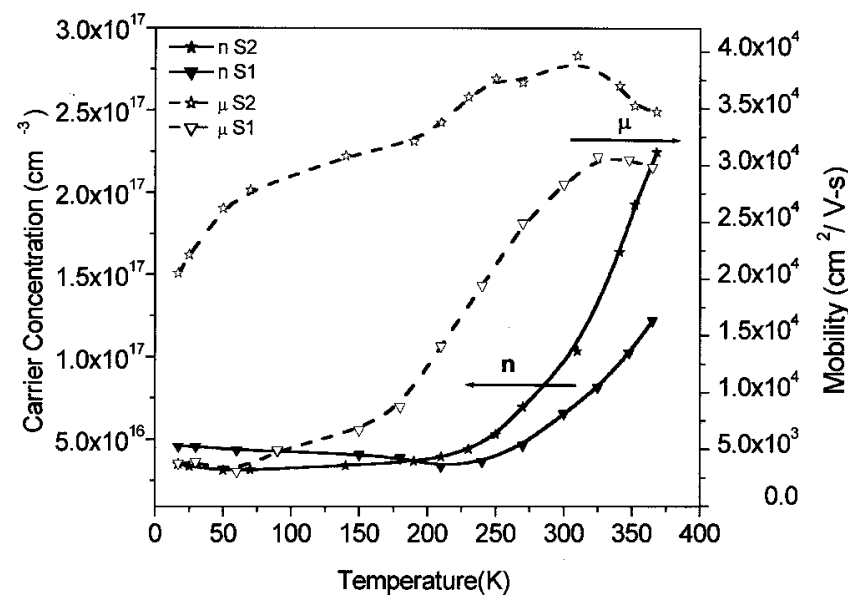

FIG. 3. Temperature dependence of the carrier concentration (closed symbols) and Hall mobility (open symbols).

too low, indicating a large number of defects formed due to lattice mismatch at the interface. These are known to limit the mobility. ${ }^{11}$ There is no observed correlation between the properties of the films with their thickness. Hence there is no prominent interface or surface conduction. Literature on MBE and MOCVD grown films also indicates that more than $2 \mu \mathrm{m}$ from the interface, the films approach bulk InSb in quality. ${ }^{10,12,17,18,22}$ The Hall data show an anomalous minimum in carrier concentration at some intermediate temperature, which has been observed previously in thin layers and has been attributed to two or three layer parallel conduction. ${ }^{20,21,23,24}$ However, since the samples are relatively thick $(3-7 \mu \mathrm{m})$ such an interpretation is not possible here. The nature and spatial distribution of dislocations along the growth direction may be a possible cause of the anomaly.

Infrared spectra in the wavelength range of 3-25 $\mu \mathrm{m}$ at $\mathrm{RT}$ and at 80 and $10 \mathrm{~K}$ are shown in Fig. 4. The onset of absorption fits very well to the relation $\alpha \sim(E-h \nu)^{1 / 2}$, indicating a direct energy gap (inset in Fig. 4). The energy gaps were measured to be $0.17,0.22$, and $0.23 \mathrm{eV}$ at RT and at 80 and $10 \mathrm{~K}$, respectively. The values and the temperature de-

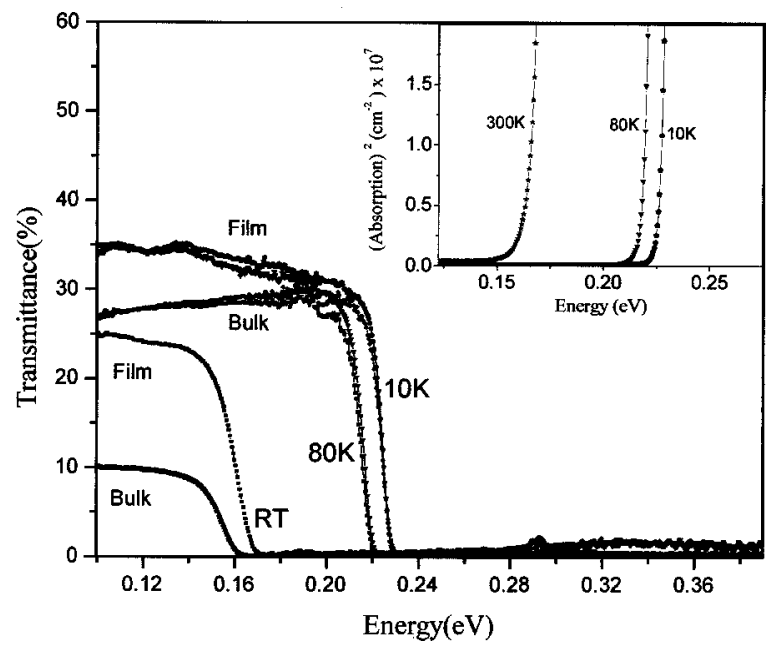

FIG. 4. Infrared spectra of bulk InSb (for comparison) and InSb/GaAs at different temperatures. The inset shows an (absorption) ${ }^{2}$ vs energy plot at different temperatures (film thickness $7 \mu \mathrm{m}$ ). pendence of the energy gap conform to standard results. ${ }^{25}$ The low temperature energy gap is within the 3.3-5.5 $\mu \mathrm{m}$ atmospheric window required for midwave infrared (MWIR) detectors.

To conclude, we have shown that InSb films of 3-7 $\mu \mathrm{m}$ thickness can be epitaxially grown on lattice-mismatched (001) GaAs substrate by liquid-phase epitaxy. The results indicate that the films are of a high quality and single crystalline with abrupt interface and high mobilities. Hence, LPE can be considered to be an inexpensive and viable alternative for industrial growth of such heterostructures, which are so important for IR detector and magnetic sensor applications.

This work was partially supported by a DTSR project and is gratefully acknowledged. The authors thank Vikram Kumar and his team at the Solid State Physics Laboratory, Delhi, for the low temperature IR data. One of the authors (V.K.D.) acknowledges CSIR, India, for awarding him a senior research fellowship.

${ }^{1}$ P. G. Kornreich, L. Walsh, J. Flattery, and S. Isa, Solid-State Electron. 29, 421 (1986).

${ }^{2}$ T. Ashley, A. B. Dean, C. T. Elliott, G. J. Pryce, A. D. Johnson, and H. Willis, Appl. Phys. Lett. 66, 481 (1995).

${ }^{3}$ J. Heremans, J. Phys. D 26, 1149 (1993).

${ }^{4}$ K. Sugiyama, J. Cryst. Growth 60, 450 (1982).

${ }^{5}$ R. Venkataraghavan, K. S. R. K. Rao, M. S. Hegde, and H. L. Bhat, Phys. Status Solidi A 163, 93 (1997).

${ }^{6}$ B. V. Rao, T. Okamoto, A. Shinmura, D. Gruznev, M. Mori, T. Tambo, and C. Tatsuyama, Appl. Surf. Sci. 159-160, 335 (2000).

${ }^{7}$ S. D. Parker, R. L. Williams, R. Droopad, R. A. Stradling, K. W. J. Barnham, S. N. Holmes, J. Laverty, C. C. Phillips, E. Skuras, R. Thomas, X. Zhang, A. Staton-Beven, and D. W. Pashley, Semicond. Sci. Technol. 4, 663 (1989).

${ }^{8}$ M. Mori, Y. Nizawa, Y. Nishi, K. Mae, T. Tambo, and C. Tatsuyama, Appl. Surf. Sci. 159-160, 328 (2000).

${ }^{9}$ S. V. Ivanov, A. A. Boudza, R. N. Kutt, N. N. Ledentsov, B. Ya Meltser, S. S. Ruvimov, S. V. Shaposhnikov, and P. S. Kop'ev, J. Cryst. Growth 156, 191 (1995).

${ }^{10}$ P. E. Thompson, J. L. Davis, J. Waterman, R. J. Wagner, D. Gammon, D. K. Gaskill, and R. Stahlbush, J. Appl. Phys. 69, 7166 (1991).

${ }^{11}$ B. S. Yoo, M. A. McKee, S. G. Kim, and E. H. Lee, Solid State Commun. 88, 447 (1993).

${ }^{12}$ D. K. Gaskill, G. T. Stauf, and N. Bottka, Appl. Phys. Lett. 58, 1905 (1991).

${ }^{13}$ M. Kumagawa, A. F. Witt, M. Lichtensteiger, and H. C. Gatos, J. Electrochem. Soc. 120, 583 (1973)

${ }^{14}$ D. E. Holmes and G. S. Kamath, J. Electron. Mater. 125, 1873 (1978).

${ }^{15}$ R. Venkataraghavan, N. K. Udayashankar, B. V. Rodrigues, K. S. R. K. Rao, and H. L. Bhat, Bull. Mater. Sci. 22, 133 (1999).

${ }^{16}$ M. Elwenspoek, J. Cryst. Growth 76, 514 (1986).

${ }^{17}$ R. M. Biefeld and G. A. Hebner, J. Cryst. Growth 109, 272 (1991).

${ }^{18}$ E. Michel, J. D. Kim, S. Javadpour, J. Xu, I. Ferguson, and M. Razeghi, Appl. Phys. Lett. 69, 215 (1996).

${ }^{19}$ J. E. Oh, P. K. Bhattacharya, Y. C. Chen, and S. Tsukamoto, J. Appl. Phys. 66, 3618 (1989).

${ }^{20}$ J. Heremans, D. L. Partin, D. T. Morelli, C. M. Thrush, G. Karczewski, and J. K. Furdyna, J. Appl. Phys. 74, 1793 (1993).

${ }^{21}$ E. Michel, H. Mohseni, J. D. Kim, J. Wojkowski, J. Sandven, J. Xu, M. Razeghi, R. Bredthauer, P. Vu, W. Mitchel, and M. Ahoujja, Appl. Phys. Lett. 71, 1071 (1997).

${ }^{22}$ X. Weng, R. S. Goldman, D. L. Partin, and J. P. Heremans, J. Appl. Phys. 88, 6276 (2000).

${ }^{23}$ C. Besikci, Y. H. Choi, R. Sudarshan, and M. Razeghi, J. Appl. Phys. 73, 5009 (1993)

${ }^{24}$ J. R. Soderstrom, M. M. Cumming, J. Y. Yao, and T. G. Andersson, Semicond. Sci. Technol. 7, 337 (1992).

${ }^{25}$ O. Madelung, Semiconductors_BasicData (Springer, Berlin, 1996). 